INSTITUT NATIONAL DE RECHERCHE EN INFORMATIQUE ET EN AUTOMATIQUE

\title{
Cerebral blood flow recorded at high sensitivity in two dimensions using high resolution optical imaging
}

\author{
Thomas Deneux - Ivo Vanzetta — Guillaume Masson — Olivier Faugeras
}

\section{$\mathbf{N}^{\circ} 5759$}

Novembre 2005

Thème BIO

\section{apport}

de recherche 



\title{
Cerebral blood flow recorded at high sensitivity in two dimensions using high resolution optical imaging
}

\author{
Thomas Deneux $^{*}$, Ivo Vanzetta ${ }^{\dagger}$, Guillaume Masson ${ }^{\dagger}$, Olivier Faugeras ${ }^{\ddagger}$ \\ Thème BIO — Systèmes biologiques \\ Projets Odyssée
}

Rapport de recherche $\mathrm{n}^{\circ} 5759$ - Novembre 2005 - 11 pages

\begin{abstract}
Knowledge about sensory-evoked blood-flow changes is essential for constraining hemodynamic response models used to interpret functional brain imaging signals, such as fMRI. Here, we extracted 2-dimensional blood-flow and its temporal modulations from highresolution optical imaging data in the awake monkey. Optical imaging allows to track moving erythrocytes (or small clusters thereof), thus providing, albeit noisy, information about their velocity in individual blood vessels, across the whole imaged area. Here, we illustrate the algorithms that allowed us to extract, at the single micro-vessel level, red blood cell (RBC) motion information from the noisy optical signals. For this purpose, we developed an algorithm that is both robust and computationally efficient, using the structure tensor, known to detect an average direction of image intensity gradient. This structure tensor tool is applied to detect trajectory directions in the spatio-temporal data. Since blood-flow modulation by the cardiac pulsation was clearly detected, our method should be applicable also to study blood-flow modulations by neuronal activity, and their spatio-temporal patterns.
\end{abstract}

Key-words: optical imaging, cerebral blood flow, optical flow, tracking, structure tensor

\footnotetext{
* thomas.deneux@ens.fr

$\dagger$ Equipe Dynamique de la Vision et de l'Action, INCM, CNRS-UMR 6193, Marseille

$\ddagger$ olivier.faugeras@sophia.inria.fr
}

\author{
Unité de recherche INRIA Sophia Antipolis \\ 2004, route des Lucioles, BP 93, 06902 Sophia Antipolis Cedex (France) \\ Téléphone : +334923877 77-Télécopie : +33492387765
}




\section{Détection d'un flux sanguin bi-dimensionnel en imagerie optique de haute résolution}

Résumé : La connaissance des variations du flux sanguin en réponse à une stimulation neuronale est essentielle pour modéliser la réponse hémodynamique observée en IRM fonctionnelle. Nous développons une méthode pour extraire des images bi-dimensionnelles du flux sanguin et ses variations dans le temps à partir de données d'imagerie optique de haute résolution acquises chez le singe éveillé. Ces données permettent de suivre le mouvement des erythrocytes (ou de petits clusters), ce qui permet de calculer la vitesse du sang dans chacun des vaisseaux, et ce dans toute la surface de l'image acquise. Nous présentons ici l'algorithme qui nous a permis d'extraire, au niveau de la micro-vasculature, l'information de mouvement des cellules de sang à partir des signaux d'imagerie optique. Cet algorithme, à la fois robuste et rapide, utilise le tenseur de structure, connu surtout pour son utilisation dans la détection de la direction principale du gradient d'intensité dans les images. Cet outil nous sert à détecter les directions des trajectoires des cellules de sang dans les données spatio-temporelles. Etant donné que nous avons pu récupérer les modulation du flux sanguin dues à la pulsation cardiaque, notre méthode doit pouvoir être appliquée à l'étude de la réponse du flux à l'activité neuronale, et à l'étude de ses variations spatiales.

Mots-clés : imagerie optique, flux sanguin, flux optique, détection de mouvement, tenseur de structure 


\section{Introduction}

Baseline blood flow and its sensory-evoked changes are commonly measured by the Laser Doppler technique. This method, however, allows only single-point measurements. Furthermore, blood flow values are integrated over a relatively large volume $\left(>1 \mathrm{~mm}^{3}\right)$, hampering the resolution of blood flow dynamics in single vessels. This is possible using 2-photon fluorescent microscopy $(1 ; 2)$, but, due to finite scanning times, this method is applicable only to one vessel at a time. Using reflection-based optical imaging, Grinvald et al. (3) succeeded to measure blood flow in large portions of the human retina. However, they reported only baseline values but no modulations. We extended this latter approach to image baseline blood flow and its modulations in the awake monkey primary visual cortex, using high-resolution optical imaging ( 15 micron/pixel, $5 \mathrm{~ms} /$ frame).

As motion detection needs to be carried out in every selected vessel and for every individual trial, we developed an algorithm that is both robust and computationally efficient. To test the method's sensitivity, our primary goal here was to detect blood-flow modulation by heart pulsation, which can be easily verified. We achieved this by using the structure tensor tool. The structure tensor determines a mean direction for intensity gradient in an image; in particular it has been used to detect edges and corners (4). The obtained blood flow signals were concordant to those recorded simultaneously by a Laser-Doppler probe, from the same piece of cortex. This validation suggests that the obtained SNR should be sufficient to resolve also blood-flow modulations known to be caused by local neuronal activation (5).

Moreover, simultaneously with the blood flow signal, here, also blood volume changes are, -so to say, "automatically"- recorded (or oximetric changes, depending on the wavelength used for imaging (6)). This allows a detailed characterization of the interplay between the various hemodynamic responses, selective for the different microvascular compartments. Such information should prove useful to adequately constrain hemodynamic models, as used to model fMRI responses.

\section{Method}

\subsection{Data preprocessing}

Blood is not a homogeneous fluid; rather, hemoglobin travels through blood vessels, packed in red blood cells $(\mathrm{RBC}) \mathrm{s}$. When the vasculature is imaged at sufficiently high resolution, these RBCs (or clusters thereof) are visible in the recorded images as small contrast changes that shift along the vasculature with time. Since, however, the large differences in reflected light intensity between blood vessels and parenchyma are much larger than the small intravascular light-intensity differences due to the presence or not of RBCs, raw images do not allow to appreciate single RBCs (one example is shown in Fig. 1, left panel). These large differences can be eliminated by normalizing for the static light intensity differences among different parts of the image, dividing on a pixel-by-pixel basis, each frame by its mean value over an acquisition sequence, and allowing thereby to "flatten the image". The contrast can

$\mathrm{RR} \mathrm{n}^{\circ} 5759$ 


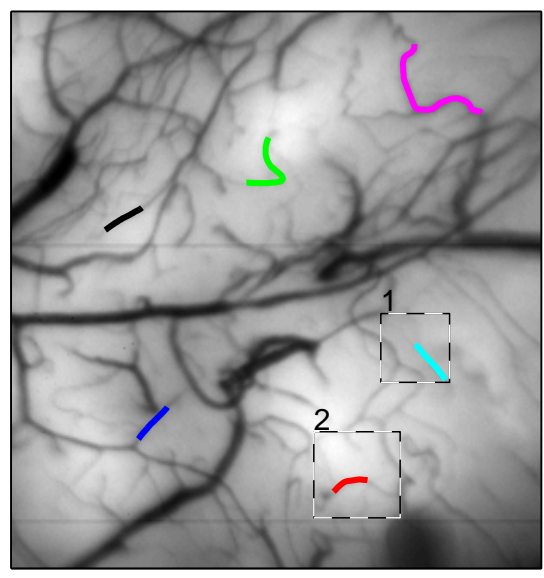

rect. $10 \mathrm{~ms}$

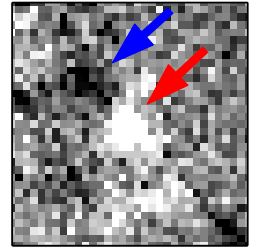

rect. $10 \mathrm{~ms}$

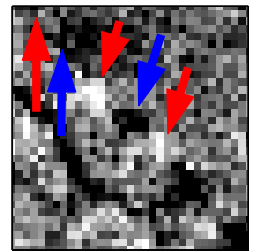

rect. $20 \mathrm{~ms}$

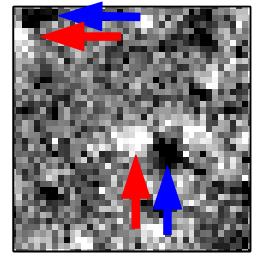

$25 \mathrm{~ms}$

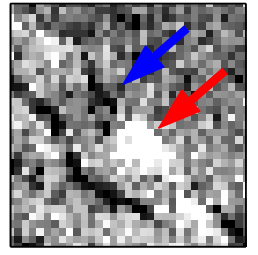

$25 \mathrm{~ms}$

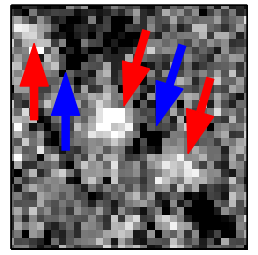

$25 \mathrm{~ms}$

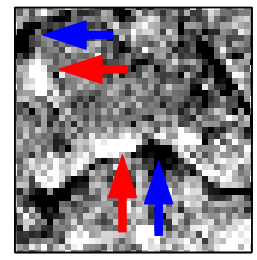

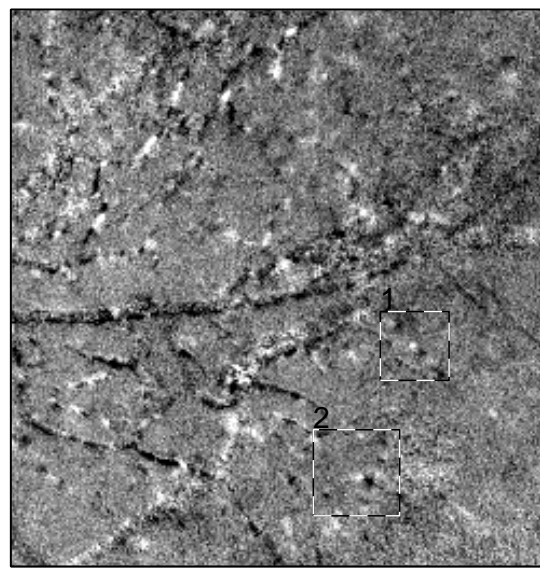

$50 \mathrm{~ms}$

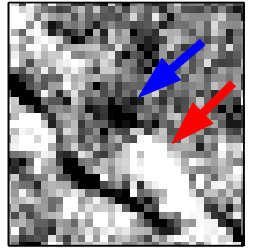

$50 \mathrm{~ms}$

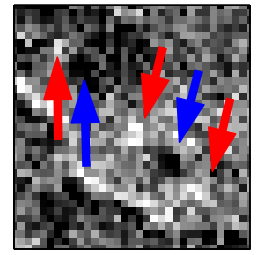

$50 \mathrm{~ms}$

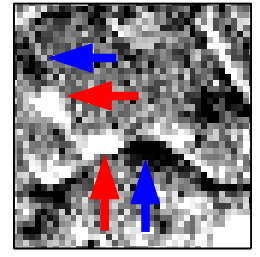

$75 \mathrm{~ms}$

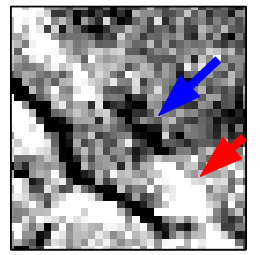

$75 \mathrm{~ms}$

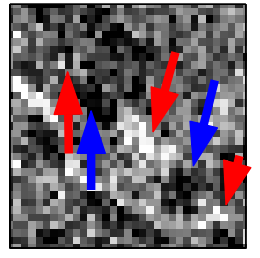

$75 \mathrm{~ms}$

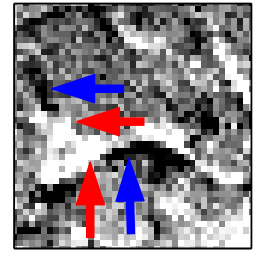

Figure 1: Top: (left) Raw image of the brain vasculature. (right) "Enhanced" image: one data frame, after division by the average of all data frames in the same trial, displayed at enhanced contrast. Black (white) dots correspond to loci of increased (decreased) local RBC concentration in the single frame with respect to average. Bottom: Zooms onto individual small vessels (rectangles in the images above). For each sequence (line), the 4 shown "enhanced" frames (time shown on top) show the motion of RCBs, or clusters thereof (arrows) through a small blood vessel. 

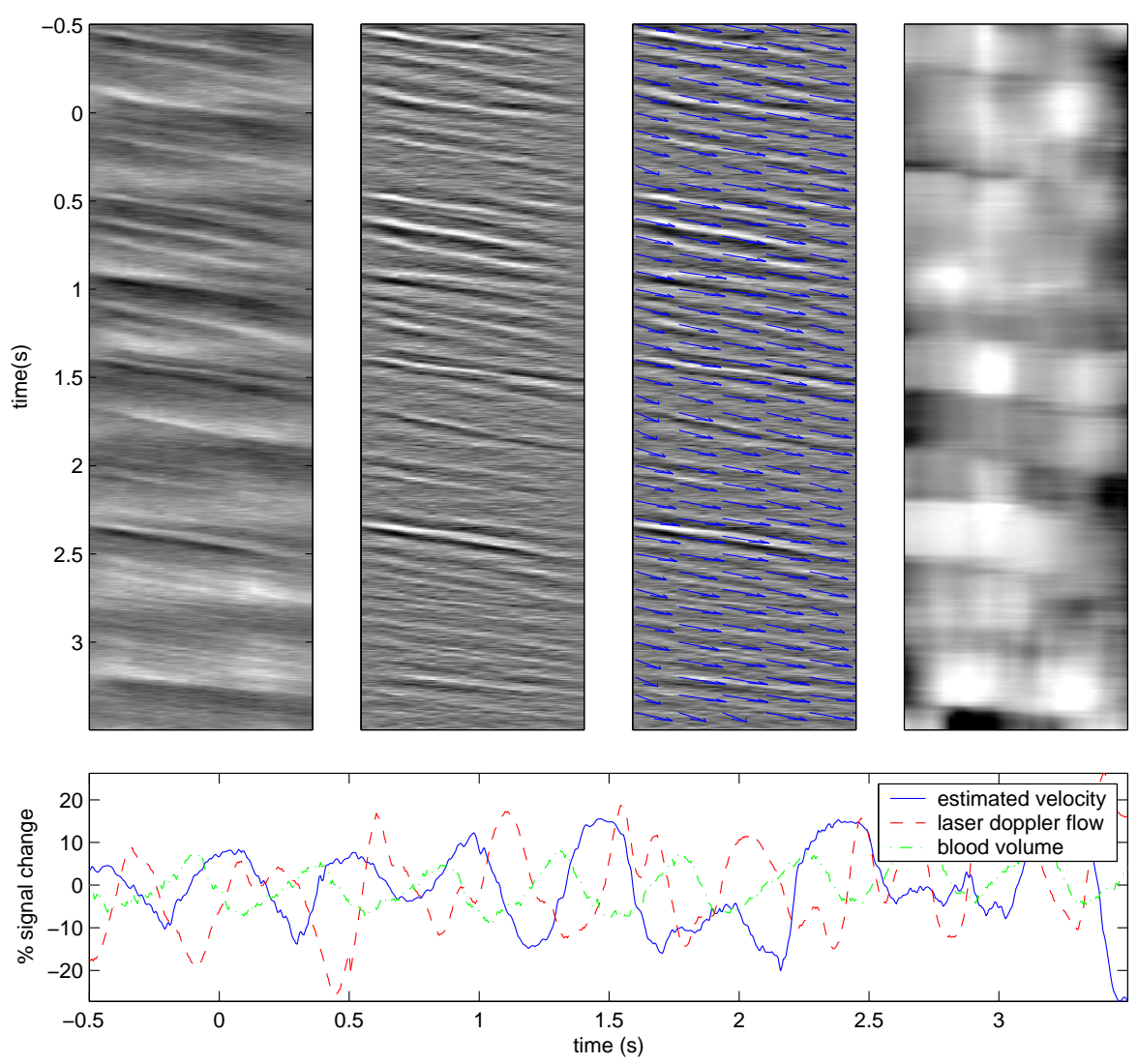

Figure 2: From left to right: Example of reflected light-intensity time course in a vessel (red in Fig. 1), for a single trial, represented as a time x space array. Second panel: high-pass filtering removed global reflected light intensity variations due to heart pulsation. Third panel: trajectory directions (arrows) found by our algorithm. Fourth panel: estimated RCBs velocity is obtained as the inverse of the trajectories' slope. Bottom: RBC velocity time course (average over all points in the vessel) is compared, for the same single trial, to Laser Doppler and blood-volume recordings: signals are concordant up to a phase shift. Here and in all other graphs the estimated RBC-velocity signal is expressed in arbitrary units (percentual change with respect to its mean) and the other signals are scaled for ease of comparison

then be largely enhanced and it becomes possible to track single clusters of RBCs moving along single vessels (Fig. 1 right panel and zooms).

Individual vessels were segmented by hand, and then light-intensity time courses in each of them were extracted from the images as $2 \mathrm{D}$ arrays $\left(n_{t}\right.$ time points by $n_{x}$ spatial points along the vessel). Figure 2 (left) shows such light-intensity time courses. The main observed feature were large horizontal "waves". Those pulsations do not result from changes in the 
RBCs' velocity. Rather, they result from the well known overall light absorption changes, due to blood-volume oscillations caused by the heart-beat $(\sim 0.5 \mathrm{~Hz}$ for our monkey, as measured independently by a pulse-oximeter).

Superimposed on those large horizontal waves, one can also observe roughly straight, oblique lines through the image: Those are the spatiotemporal trajectories of RBCs in the vessel (they move right when time increases), which contain the RBCs' motion information. The slope of these lines is indeed directly linked to RCB velocity: if a hemoglobin packet moves from position $x$ to position $x^{\prime}$ between times $t$ and $t+1$, then its velocity along the vessel direction is $x^{\prime}-x$. The higher the speed, the more the lines will approach horizontality. These trajectories were then enhanced by 2-D high-pass filtering of the image (thus with respect to both time and space), to remove the global absorptive effects of heart pulsation (Fig. 2, second panel).

\subsection{Detecting hemoglobin trajectories}

$\mathrm{RBC}$ speed determination is achieved by resolving the RBC's trajectories in each individual vessel, i.e. by determining the lines' directions in 2D images as in Figure 2 (third panel). Let's notate $I$ the image representing intensity time courses in the vessel, $I_{t}$ and $I_{x}$ its derivatives with respect to time and space. Then the gradient vector at point $(x, t)$ is $\left(I_{x}(x, t)\right.$, $\left.I_{t}(x, t)\right)$, and the direction of trajectories lines is its orthogonal vector $\left(-I_{t}(x, t), I_{x}(x, t)\right)$. $\mathrm{RBC}$ speed is then given by the inverse of this trajectories' slope, $v(x, t)=-I_{t}(x, t) / I_{x}(x, t)$.

Formally, we prove this formula by writing the optical flow equation (7), which states that reflected light intensity does not change along particles trajectories: if we notate $x(t)$ the position of a given particle at time $t$, we have

$$
\begin{aligned}
\frac{\partial}{\partial t} I(x(t), t) & =0 \\
v I_{x}+I_{t} & =0 \\
v & =-I_{t} / I_{x} .
\end{aligned}
$$

This formula for RBC speed, however, cannot be applied directly because of noise in the image. It is necessary to stabilize the algorithm by using a neighborhood averaging. The idea is to find an average direction of trajectories over a small neighbourhood (we used a neighborhood of about 30 time points by 5 voxels length), which can be achieved with the structure tensor.

Let us define the following degenerate symmetric matrix:

$$
A(x, t)=\left(\begin{array}{cc}
I_{x}^{2}(x, t) & I_{x}(x, t) I_{t}(x, t) \\
I_{x} . I_{t}(x, t) & I_{t}^{2}(x, t)
\end{array}\right) .
$$

The first eigenvector of $A$ is the gradient direction $\left(I_{x}, I_{t}\right)$ and its second eigenvector (with zero eigenvalue) is our trajectories' direction $\left(-I_{t}, I_{x}\right)$. The structure tensor is defined by averaging matrix A over a small neighborhood

$$
S(x, t)=\sum_{\left(x^{\prime}, t^{\prime}\right) \in \mathcal{V}(x, t)} A\left(x^{\prime}, t^{\prime}\right) .
$$



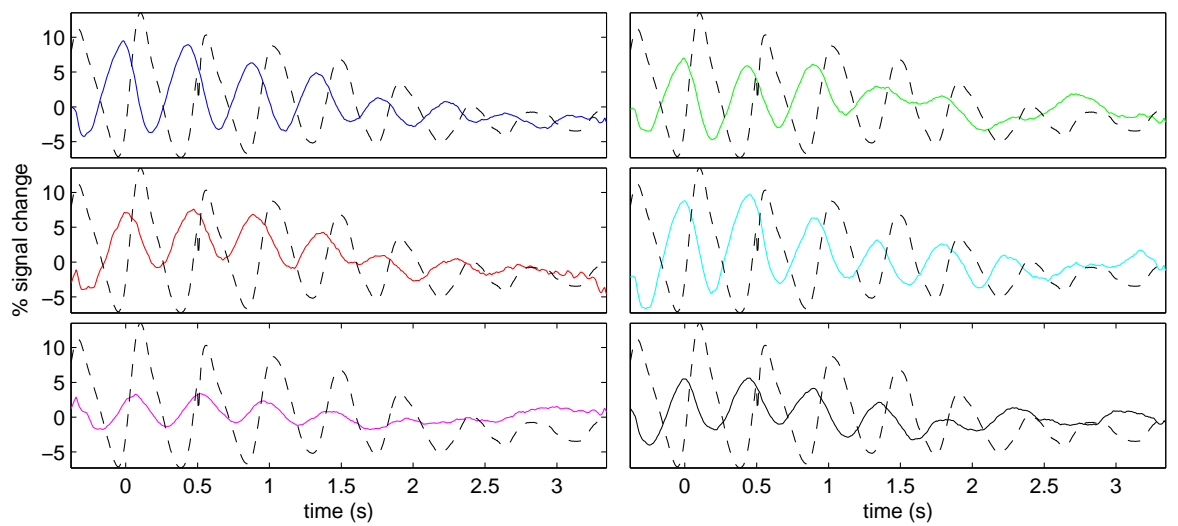

Figure 3: Estimated RCBs velocities in five vessels (same color-codes for time courses as vessels in Fig. 1) and comparison with Laser Doppler (dashed line) measure. Time courses are averaged on all trial presentations. Heart pulsation is correctly recovered. Differences in phase between vessels indicate delays in blood transfer. The amplitude decreases because inter-trial heart-beat synchronization deteriorates progressively.

Then the first eigenvector of $S$ gives the direction in which image intensity varies the most ; the second one gives the direction in which image intensity varies the less, which is actually the direction we are looking for, and the inverse of its slope is the estimated RCBs velocity.

\subsection{Vessels coregistration}

The flow estimation was carried out for each individual trial. The vessels have been drawn by hand on one raw image (using Fig. 1, left panel). However, it happens that, because of the animal's movements, the whole vasculature shifts slightly from one trial to the next. To average, it is thus necessary to register these rigid motions. This was accomplished by finding the isometry (a rotation plus a translation) that minimizes the differences between vasculature images:

$$
(\hat{\theta}, \hat{\mathbf{t}})=\underset{\theta, \mathbf{t}}{\operatorname{argmin}}\left\|I_{2}-\mathcal{T}_{\mathbf{t}} \circ \mathcal{R}_{\theta}\left(I_{1}\right)\right\|^{2}
$$

\subsection{Results}

To increase SNR of the recovered heart response, data were averaged over trials $(n=144$; data acquisition was synchronized with heartbeat). Figure 3 shows the obtained velocity time courses for several vessels, well matching with the simultaneous Laser-Doppler measurements.

$\mathrm{RR} \mathrm{n}^{\circ} 5759$ 


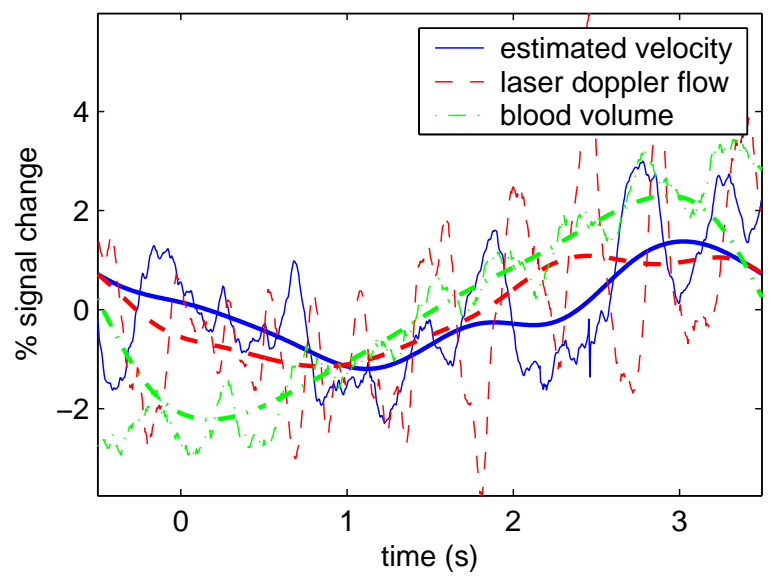

Figure 4: Blood flow fluctuations superimposed onto cardiac pulsation. Two RBC-velocity time courses were averaged over two large independent datasets (sixty trials per dataset), and then the ratio was calculated as a function of time (dashed lines), representing the difference between the two averaged signals, expressed in fractional units of their mean (blood-flow from Laser Doppler and blood volume signals were scaled to ease comparison). Solid lines represent temporally low-passed traces $(300 \mathrm{~ms})$ to reduce the noise resulting from the division.

Finally, we wanted to test the sensitivity of our method with respect to small, non-heartbeat-related blood-flow changes (Fig. 4). First, we divided the trials into two independent sets. We then averaged the velocity estimations over each of the two sets. We then calculated the ratio between those two averaged signals, as is customary done to extract sensoryevoked hemodynamic responses $(5 ; 6)$, and compared it to (i) the same ratio obtained from the simultaneously acquired Laser Doppler and from (ii) blood volume recordings. The similarity between all the 3 obtained time courses shows that our algorithm can also capture signal fluctuations that are superimposed on heart pulsation, such as those resulting from neuronal activity, evoked by sensory stimulation.

\subsection{Experimental setup}

Preparation: Experiments were performed on an awake adult male macaque monkey (M. fascicularis, $6-7 \mathrm{Kg}$ ), with a transparent cranial window chronically implanted onto a $\sim 2 \mathrm{x} 2 \mathrm{~cm}$ aperture in the skull above the V1/V2 border, at about $2.5^{\circ}$ eccentricity of the visual field. For a detailed description of all surgical and maintenance procedures see (8).

Experimental paradigm: Data acquisition was synchronized with the heartbeat, recorded independently by a pulse-oximeter. A trial started when the monkey began fixating on a fixation point $\left(0.08-0.1^{\circ}\right)$, displayed on a CRT screen. The monkey had to continue to fixate until the fixation point disappeared, for a total fixation period of about 4s. An isoluminant uniform, grey screen, was shown between the trials. Inter-trial interval was at least 10s. To avoid visually-evoked noise, data from trials where the monkey broke fixation were rejected. 
Optical recordings: We imaged 3.5x5 millimeters of cortex, continuously over 4 seconds, illuminating at a wavelength that is isosbestic for oxy-and deoxy-hemoglobin $(570 \mathrm{~nm})$ to avoid oximetric artifacts. Images were acquired at $200 \mathrm{~Hz}$ using a commercial, CCD-based, imaging system (Imager 3001, Germantown, USA).

Laser-Doppler recordings: We used a Periflux 5001 (Perimed, Stockholm; wavelength of $780 \mathrm{~nm}$ ) laser Doppler flowmeter to which a needle probe (Perimed PROBE 411; outer diameter, 450 micron; fiber separation, 150 micron - estimated sample volume: $\sim 1 \mathrm{~mm}^{3}$ ) was attached. The probe was positioned $\sim 1 \mathrm{~mm}$ from the brain surface.

\section{Discussion}

The main challenges to the estimation of RCBs' motion in the brain vasculature are low SNR and possible artifacts. To achieve sufficient SNR, high temporal resolution acquisition was needed $(200 \mathrm{~Hz}$ sampling was necessary to get evident trajectories), followed by adequate data preprocessing (imposing motion directions by a priori vessel-segmentation, registering movements between trials, high-pass filtering to remove heart pulsation effects).

Concerning the velocity estimation, it is of primary importance to find out which quantity can be spatially averaged to handle high frequency noises: smoothing the data directly would make the trajectories disappear; estimating blood velocity directly from the optical flow equation (1) and then smoothing the estimations would lead to a non-sense, because of the $I_{x}$ division in (1), that could be zero and thus diverge. The employed structure tensor presents the advantage of being a quantity that represents the local orientations structure, and that can be averaged in a neighborhood in a meaningful way.

Nevertheless, the algorithm performs poorly when several directions appear in the spatiotemporal arrays, due to remaining heart beat effects, fast vessel movement under blood pressure, or superposition with other vessels.... An average direction is then computed, instead of choosing the one corresponding to the true RCB's motion. Other algorithms, for example using wavelets methods, might be able to overcome this problem.

\section{Applications and conclusion}

As opposed to standard methods providing only point-like blood flow measurements, our method allows to determine blood flow and its modulations across a 2 dimensional image. This should prove useful to study the neurally-evoked blood flow responses, as those resulting from sensory stimuli, and underlying PET and fMRI brain imaging signals. Furthermore, the resolution of single vessels allows to study blood-flow in different vascular compartments. Together and simultaneously with RBCs velocity, blood-volume and/or oximetric changes (6) are also "automatically" recorded. This allows to characterize those different physiological responses at the single-vessel level, for many vessels and across different functional domains, thus providing high-resolution functional "blood-flow maps" in addition to those which are

$\mathrm{RR} \mathrm{n}^{\circ} 5759$ 
routinely obtained by blood volume and oximetry (6), as well as important constraints for hemodynamic response models, as those used in modern functional brain imaging techniques.

\section{Acknowledgements}

I. Vanzetta has been supported by a fellowship from the Fondation pour la Recherche Médicale.

\section{References}

[1] David Kleinfeld, Partha P. Mitra, Fritjog Helmchen, and Winfried Denk, "Fluctuations and stimulus-induced changes in blood flow observed in individual capillaries in layers 2 through 4 of rat neocortex," PNAS USA, vol. 95, pp. 15741-15746, 1998.

[2] Emmanuelle Chaigneau, Martin Oheim, Etienne Audinat, and Serge Charpak, "Twophoton imaging of capillary blood flow in olfactory bulb glomeruli," Proc Natl Acad Sci U S A, vol. 100, no. 22, pp. 13081-13086, Oct 2003.

[3] Amiram Grinvald, Tobias Bonhoeffer, Ivo Vanzetta, Ayala Pollack, Eyal Aloni, Ron Ofri, and Darin Nelson, "High-resolution functional optical imaging: from the neocortex to the eye," Ophthalmol Clin N Am, vol. 17, pp. 53-67, 2004.

[4] Ullrich Köthe, "Edge and junction detection with an improved structure tensor," 2003, pp. 25-32.

[5] M Jones, J Berwick, D Johnston, and J Mayhew, "Concurrent optical imaging spectroscopy and laser-Doppler flowmetry: the relationship between blood flow, oxygenation, and volume in rodent barrel cortex," Neuroimage, vol. 13, no. 6 Pt 1, pp. 1002-1015, Jun 2001.

[6] R D Frostig, E E Lieke, D Y Ts'o, and A Grinvald, "Cortical functional architecture and local coupling between neuronal activity and the microcirculation revealed by in vivo high-resolution optical imaging of intrinsic signals," Proc Natl Acad Sci U S A, vol. 87, no. 16, pp. 6082-6086, Aug 1990.

[7] T. Brox, A. Bruhn, N. Papenberg, and J. Weickert, "High accuracy optical flow estimation based on a theory for warping," in Proceedings of the 8th European Conference on Computer Vision, Prague, Czech Republic, Springer-Verlag., Ed., 2004.

[8] Amos Arieli, Amiram Grinvald, and Hamutal Slovin, "Dural substitute for long-term imaging of cortical activity in behaving monkeys and its clinical implications," Journal of Neuroscience Methods, vol. 114, pp. 119-133, 2002. 


\section{Contents}

1 Introduction $\quad 3$

2 Method $\quad 3$

2.1 Data preprocessing ... . . . . . . . . . . . . . . 3

2.2 Detecting hemoglobin trajectories . . . . . . . . . . . . . . . . . . . . . . . . . . . . .

2.3 Vessels coregistration . . . . . . . . . . . . . . . . . . . 7

2.4 Results.............................. . . . 7

2.5 Experimental setup . . . . . . . . . . . . . . . . . . . 8

3 Discussion $\quad 9$

4 Applications and conclusion $\quad 9$

$\mathrm{RR} \mathrm{n}^{\circ} 5759$ 


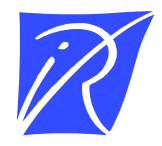

\section{Unité de recherche INRIA Sophia Antipolis 2004, route des Lucioles - BP 93 - 06902 Sophia Antipolis Cedex (France)}

Unité de recherche INRIA Futurs : Parc Club Orsay Université - ZAC des Vignes 4, rue Jacques Monod - 91893 ORSAY Cedex (France)

Unité de recherche INRIA Lorraine : LORIA, Technopôle de Nancy-Brabois - Campus scientifique 615, rue du Jardin Botanique - BP 101 - 54602 Villers-lès-Nancy Cedex (France)

Unité de recherche INRIA Rennes : IRISA, Campus universitaire de Beaulieu - 35042 Rennes Cedex (France)

Unité de recherche INRIA Rhône-Alpes : 655, avenue de l'Europe - 38334 Montbonnot Saint-Ismier (France)

Unité de recherche INRIA Rocquencourt : Domaine de Voluceau - Rocquencourt - BP 105 - 78153 Le Chesnay Cedex (France)

INRIA - Domaine de Voluceau - Rocquencourt, BP 105 - 78153 Le Chesnay Cedex (France)

http://www.inria.fr

ISSN 0249-6399 